Venina Isabel Poço Viana Leme de Barros ${ }^{1}$

ANa Maria Kondo lgal

Marina de PAula ANDRES ${ }^{2}$

Rossana Pulcaineul Viera Francisco 3

MARCEO ZUGAiB ${ }^{4}$

Artigo Original

\author{
Palavras-chave \\ Morte fetal \\ Trombofilia \\ Complicações na gravidez \\ Keywords \\ Fetal death \\ Thrombophilia \\ Pregnancy complications
}

\section{Resultados gestacionais e trombofilias em mulheres com história de óbito fetal de repetição}

\section{Pregnancy outcome and thrombophilia of women with recurrent fetal death}

\section{Resumo}

OBJETIVO: Descrever desfechos obstétricos e frequência de trombofilias em gestantes com óbito fetal de repetição após a 20 semana de gravidez. MÉTODOS: Avaliação de desfechos obstétricos em uma série de casos de gestantes com óbito fetal de repetição após a 20ª semana de gestação, acompanhadas de 2001 a 2013 . A atividade de antitrombina, atividade da proteína $\mathrm{C}$ e S, presença de fator $\mathrm{V}$ de Leiden, presença da mutação do gene de protrombina e presença de síndrome antifosfolípide foram avaliadas nessas pacientes. RESULTADOS: Foram incluídas 20 pacientes que tinham óbito fetal de repetição. Trombofilias foram encontradas em 11 delas, sendo 7 diagnosticadas como síndrome antifosfolípide, 3 como deficiência de proteína S e 1 como mutação do gene da protrombina. Todas foram tratadas com heparina subcutânea (heparina não fracionada ou enoxaparina) e 14 delas com ácido acetilsalicílico (AAS) durante toda a gestação. Complicações obstétricas ocorreram em 15 pacientes e incluíram: restrição de crescimento fetal intrauterino (25\%), placenta prévia (15\%), índice de líquido amniótico diminuído (25\%), pré-eclâmpsia

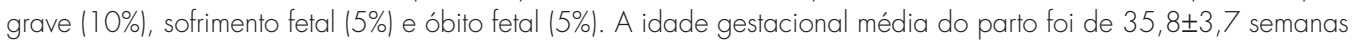

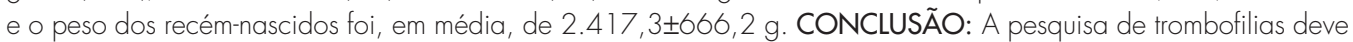
ser realizada em todas as gestantes com óbitos fetais de repetição após a $20^{a}$ semana de gestação, como forma de identificar possiveis fatores causas passíveis de tratamento.

\section{Abstract}

PURPOSE: To evaluate pregnancy outcome and thrombophilia frequency in women with recurrent fetal death. METHODS: Evaluation of obstetric outcomes in a retrospective cohort of pregnant women with recurrent stillbirth after the 20, th week, from 2001 to 2013 . Antithrombin activity, protein C and S activity, factor V Leiden, prothrombin gene mutation and antiphospholipid syndrome were analyzed. RESULTS: We included 20 patients who had recurrent fetal death. Thrombophilia were found in 11 of them, 7 diagnosed with antiphospholipid syndrome, 3 with protein S deficiency and 1 with prothrombin gene mutation. All of them were treated with subcutaneous heparin (unfractionated heparin or enoxaparina) and 14 of them with acetylsalicylic acid (AAS) during pregnancy. Obstetric complications occurred in 15 patients and included: intrauterine fetal growth restriction (25\%), placenta previa (15\%), reduced amniotic fluid index $(25 \%)$, severe preeclampsia $(10 \%)$, fetal distress $(5 \%)$, and stillbirth $(5 \%)$. The mean gestational age at delivery

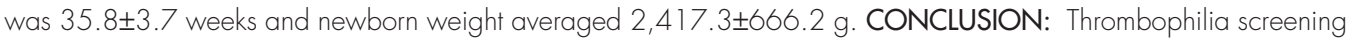
should be performed in all pregnant women with recurrent fetal death after the $20^{\text {th }}$ week as a way to identify possible causal factors suitable for treatment.
Correspondêncio Venina Isabel Poço Viana Leme de Barros Avenida Dr. Enéas de Carvalho Aguiar, 255 CEP: 05403-000 São Paulo (SP), Brasil

Recebido 07/10/2013
Departamento de Obstetrícia do Hospital das Clínicas da Faculdade de Medicina da Universidade de São Paulo - USP - São Paulo (SP), Brasil. 'Ambulatório de Tromboses e Gestação, Departamento de Obstetrícia, Hospital das Clínicas, Faculdade de Medicina, Universidade de São Paulo - USP - São Paulo (SP), Brasil.

2Programa de Residência em Ginecologia e Obstetrícia, Hospital das Clínicas, Faculdade de Medicina, Universidade de São Paulo USP - São Paulo (SP), Brasil.

${ }^{3}$ Disciplina de Obstetrícia, Faculdade de Medicina, Universidade de São Paulo - USP - São Paulo (SP), Brasil.

${ }^{4}$ Disciplina de Obstetrícia e Ginecologia, Faculdade de Medicina, Universidade de São Paulo - USP - São Paulo (SP), Brasil.

Conflito de interesses: não há. 
Introdução

A predisposição à trombose, conhecida como trombofilia, encontra-se potencializada na gestação e no puerpério pelas próprias características pró-coagulantes desses estados ${ }^{1,2}$. As razões para esse aumento são multifatoriais e incluem: aumento dos níveis hormonais, diminuição do fluxo venoso nos membros inferiores, fatores mecânicos (como aumento da pressão nos vasos pélvicos pela compressão uterina), alterações hemostáticas, aumento de fatores pró-coagulantes, como fator VII, fator VIII, fator X, fibrinogênio, e diminuição da porção livre da proteína $S^{1}$.

As trombofilias podem ser classificadas em adquiridas e hereditárias e afetam aproximadamente $15 \%$ da população geral. As trombofilias hereditárias incluem deficiências das proteínas anticoagulantes (proteína C, proteína S e antitrombina) e mutações nos genes codificadores das proteínas pró-coagulantes (fator $\mathrm{V}$ e protrombina) ${ }^{3}$.

Tanto a trombofilia adquirida quanto a hereditária podem ser identificadas em 20 a $50 \%$ das mulheres que apresentaram um evento tromboembólico durante a gestação ou pós-parto ${ }^{4}$. Além disso, sua associação com a gestação tem sido cada vez mais estudada como causa identificável de resultados adversos gestacionais, como trombose placentária, formas graves de hipertensão gestacional, descolamento prematuro de placenta, restrição de crescimento fetal intrauterino e óbito fetal ${ }^{1,5-8}$.

Essas complicações obstétricas constituem $75 \%$ das causas de morbidade e mortalidade neonatal e sua prevalência chega a aproximadamente 8\% nos Estados Unidos, totalizando 320 mil gestações por ano. Quaisquer dessas complicações em uma gestação anterior constituem forte preditor de recorrência em gestação futura ${ }^{9,10}$.

Dentre os diversos resultados perinatais adversos, encontra-se o óbito fetal, que é definido pela morte do produto conceptual, antes de sua expulsão ou extração completa do organismo materno. A maioria dos autores considera o óbito fetal aquele ocorrido posteriormente à $20^{a}$ semana de gestação ou combinações de idade gestacional e peso do produto conceptual igual ou superior a $500 \mathrm{~g}^{11}$. Em razão da diversidade dos conceitos adotados, a incidência de óbito fetal relatada na literatura é variável e inferior aos valores reais por causa de subnotificação ${ }^{12}$.

No Brasil, em casuística do Hospital das Clínicas da Faculdade de Medicina da Universidade de São Paulo, de janeiro de 1978 a dezembro de 1982, a incidência foi de 32,7 para cada 1.000 nascimentos, utilizando como critério idades gestacionais após a $20^{a}$ semana ${ }^{11}$.

De 1993 a 1998, foi encontrado um total de 526 casos entre 11.733 partos, perfazendo uma incidência de 1,6 para cada 1.000 nascimentos. Nesse estudo, 13\% das pacientes apresentavam dois ou mais óbitos fetais anteriores. Qualquer doença acompanhada de insuficiência placentária durante a gestação pode, em última instância, ser causa desse evento. Das causas de óbito fetal, $12 \%$ não são identificadas, $15 \%$ são atribuídas a complicações relacionadas ao cordão umbilical e placenta, $12 \%$ a malformações fetais, $23 \%$ a insuficiência placentária e $12 \%$ a outras causas ${ }^{13}$.

A relação entre a trombofilia adquirida, representada pela síndrome antifosfolípide, e resultados gestacionais adversos repetidos é bem estabelecida na literatura. A etiologia envolve lesão direta das células trofoblásticas e tromboses intraplacentárias, que levam a manifestações fetais de hipóxia e insuficiência placentária, como restrição de crescimento fetal intrauterino, oligohidrâmnio e alterações de dopplervelocimetria fetal ${ }^{14}$.

Uma meta-análise de 2003 incluiu 31 estudos que avaliaram pacientes com óbito fetal ${ }^{15}$. Os estudos foram heterogêneos quanto aos resultados e aos critérios de definição de óbito fetal; no entanto, o óbito fetal recorrente após a $22^{\mathrm{a}}$ semana de gestação foi relacionado à presença do fator $\mathrm{V}$ de Leiden, protrombina mutante e deficiência de proteína $S$, com aumento no risco em até 7,8 vezes.

O presente estudo visou descrever características, frequência de trombofilia e desfechos obstétricos em gestantes com óbito fetal de repetição após a $20^{\mathrm{a}}$ semana.

\section{Métodos}

O estudo foi submetido à aprovação da Comissão de Análise de Projetos de Pesquisa do Hospital das Clínicas da Faculdade de Medicina da Universidade de São Paulo (HCFMUSP) sob o número CIAPP 308/11.

Foram avaliadas retrospectivamente informações sobre gestantes com diagnóstico de trombose e/ou trombofilias na gestação, com seguimento pré-natal no ambulatório do Setor de Tromboses e Gestação da Clínica Obstétrica do HCFMUSP, no período de janeiro de 2001 a julho de 2013.

A coleta de dados foi feita a partir da análise do banco informatizado do Setor de Tromboses e Gestação, do Sistema de Enfermaria e do Sistema de Laudos da Obstetrícia e Ginecologia da Clínica Obstétrica do HCFMUSP, para avaliação das características das pacientes. Esses bancos apresentam informações preenchidas de forma prospectiva durante o atendimento pré-natal, internações hospitalares e puerpério. Os prontuários das pacientes selecionadas e de seus recém-nascidos foram consultados para coleta de informações que não constassem nos bancos de dados anteriormente citados.

Os critérios de inclusão foram: gestantes com antecedente de dois ou mais óbitos fetais e idade gestacional maior ou igual a $20^{\mathrm{a}}$ semana de gestação, confirmado pela ultrassonografia de primeiro trimestre. Os critérios de 
exclusão foram: presença de malformações fetais e gestações múltiplas.

Foram coletadas amostras de sangue periférico das gestantes no início do pré-natal (até a $12^{\mathrm{a}}$ semana de gestação) e confirmados no puerpério para a avaliação de presença de trombofilias genéticas ou adquiridas. Foram consideradas portadoras de trombofilias genéticas todas as gestantes que apresentaram resultados compatíveis com deficiência de proteína $\mathrm{C}$, deficiência de proteína $\mathrm{S}$, deficiência de antitrombina, presença de mutação no fator $\mathrm{V}$ de Leiden (heterozigoto e homozigoto afetado) ou presença do gene mutante da protrombina (fator II). Foram consideradas portadoras de anticorpos antifosfolípides todas as pacientes que obtiveram quaisquer resultados reagentes para anticardiolipina $\operatorname{IgM} / \mathrm{IgG}$, anticoagulante lúpico e/ou anti- $\beta_{2}$ microglobulina.

O diagnóstico de Síndrome Antifosfolípide foi baseado em critérios diagnósticos estritos e bem definidos, que obedecem à normatização da Sociedade Internacional de Trombose e Hemostasia ${ }^{16}$.

Os resultados dos exames laboratoriais foram obtidos do sistema de informática do Laboratório Central do HCFMUSP (disponível online pelo site www.phcnet.usp. br). As concentrações dos anticorpos anticardiolipina IgG e IgM foram obtidas pelo método ELISA (valor de corte de $21 \mathrm{GPL} / \mathrm{mL}$ para ambos) e anticoagulante lúpico, de acordo com a literatura ${ }^{16}$.

$A$ avaliação da presença do fator $\mathrm{V}$ de Leiden foi obtida por amplificação por PCR e tratamento por enzima específica ${ }^{17}$ e classificado como ausente, presente em forma homozigota ou presente em forma heterozigota. A mutação da protrombina foi obtida pelo método de Bertina ${ }^{18}$ e classificada como ausente, presente em forma homozigota ou presente em forma heterozigota. A atividade de antitrombina foi avaliada pelo método amidolítico (valor de referência de 79 a 131\%). A atividade de proteína $\mathrm{S}$ foi avaliada pelo método cronométrico (valor de referência de 55 a 160\%). A atividade da proteína $\mathrm{C}$ foi avaliada por amidolítico com substrato cromogênico (valor de referência de 64 a 128\%).

\section{Análise estatística}

Todas as variáveis anteriormente citadas foram submetidas a estudos comparativos. Foram utilizados os testes do $\chi^{2}$ e exato Fisher para as variáveis classificatórias, comparando a presença ou não de trombofilias e os resultados obstétricos encontrados. A probabilidade de significância adotada foi de 0,15.

\section{Resultados}

Das pacientes acompanhadas no pré-natal do HCFMUSP, 527 mulheres em 585 gestações pertenciam ao Setor de Tromboses e Gestação. Foram consultados seus respectivos prontuários eletrônicos do sistema de internação e incluídas 20 pacientes de acordo com os critérios de inclusão e exclusão. As informações não estavam disponíveis em 7 pacientes $(1,3 \%)$.

A frequência de óbito fetal recorrente entre as pacientes acompanhadas no Setor de Tromboses e Gestação foi $3,7 \%$. Foram encontrados 2 óbitos fetais em 17 pacientes e 3 óbitos fetais em 3 pacientes.

As características clínicas e antecedentes pessoais das pacientes incluídas estão resumidas na Tabela 1 . A média de idade das pacientes foi de 30,6士4,6 anos, com mediana de 30,5 anos e variação de 18 a 37 anos de idade. No total, 5/20 pacientes (25\%) apresentavam índice de massa corpórea maior ou igual a 30 no primeiro trimestre.

Quanto à paridade, a média foi de 5,4士1,8 gestações, variando de 3 a 9 gestações. Foram encontrados 2 ou mais abortamentos anteriores em $20 \%$ das pacientes e antecedente de descolamento prematuro de placenta em $1,5 \%$. A média de filhos vivos foi de $0,8 \pm 0,7$, variando de 0 a 2 filhos vivos.

O perfil de trombofilias mostrou alteração em 11 das 20 pacientes incluídas, sendo 7 diagnosticadas como síndrome antifosfolípide, 3 como deficiência de proteína $\mathrm{S}$ e 1 como mutação da protrombina na forma heterozigota (Tabela 2).

As 20 pacientes incluídas tiveram seguimento regular de pré-natal. Complicações obstétricas nas gestações índices ocorreram em 15 das 20 pacientes incluídas e foram semelhantes entre aquelas com e sem trombofilias (7 versus 8 pacientes, respectivamente). Dentre elas estão: restrição de crescimento fetal intrauterino $(5 / 20-25 \%)$, placenta prévia $(3 / 20-15 \%)$, índice de líquido amniótico diminuído $(4 / 20$ - 20\%), pré-eclâmpsia superajuntada $(2 / 20-10 \%)$, sofrimento fetal $(1 / 20-5 \%)$, óbito fetal $(1 / 20-5 \%)$ e diabetes gestacional $(1 / 20-5 \%)$.

Tabela 1. Características das gestantes com óbito fetal de repetição após a 20 semana de gravidez $(n=20)$

\begin{tabular}{|c|c|c|}
\hline Características & \multicolumn{2}{|c|}{$\begin{array}{c}\text { Valor } \\
\text { Média } \pm D P\end{array}$} \\
\hline Idade (anos) & \multicolumn{2}{|c|}{$30,6 \pm 4,6$} \\
\hline IMC inicial $\left(\mathrm{kg} / \mathrm{m}^{2}\right)$ & \multicolumn{2}{|c|}{$26,7 \pm 4,6$} \\
\hline Número de gestações & \multicolumn{2}{|c|}{$5,4 \pm 1,8$} \\
\hline Abortamentos anteriores & \multicolumn{2}{|c|}{$1,7 \pm 1,5$} \\
\hline Número de partos & \multicolumn{2}{|c|}{$2,9 \pm 1,2$} \\
\hline Filhos vivos & \multicolumn{2}{|c|}{$0,8 \pm 0,7$} \\
\hline Comorbidades & $n$ & $\%$ \\
\hline Anemia falciforme & 0 & - \\
\hline Diabetes mellitus tipo 2 & 2 & 10 \\
\hline Episódios tromboembólicos & 3 & 15 \\
\hline Hipertensão arterial & 7 & 35 \\
\hline Lúpus eritematoso sistêmico & 1 & 10 \\
\hline Tabagismo & 3 & 15 \\
\hline
\end{tabular}


Tabela 2. Desfechos obstétricos de pacientes com óbitos fetais de repetição, com e sem trombofilias

\begin{tabular}{lcccc}
\hline & \multicolumn{2}{c}{$\begin{array}{c}\text { Pacientes sem } \\
\text { trombofilias }(\mathrm{n}=9)\end{array}$} & \multicolumn{2}{c}{$\begin{array}{c}\text { Pacientes com } \\
\text { trombofilias }(\mathrm{n}=1 \mathrm{1})\end{array}$} \\
\cline { 2 - 5 } & $\mathrm{n}$ & $\%$ & $\mathrm{n}$ & $\%$ \\
\hline Complicações obstétricas & 8 & 88,8 & 7 & 63,3 \\
CIUR & 3 & 33,3 & 2 & 18,2 \\
ILA reduzido & 3 & 33,3 & 1 & 9 \\
Óbiło fetal & 0 & 0 & 1 & 9 \\
Infartos/hematomas placentários & 3 & 33,3 & 5 & 45,4 \\
Peso ao nascer (g) & \multicolumn{2}{c}{$2.540,0$} & \multicolumn{2}{c}{$2.316,9$} \\
Idade gestacional média (semanas) & 36 & $5 / 7$ & 35 & $3 / 7$ \\
\hline
\end{tabular}

CIUR: crescimento intrauterino restrito; ILA: índice de líquido amniótico.

Os resultados obstétricos e perinatais estão resumidos na Tabela 2. A idade gestacional média do parto foi de $36,6 \pm 0,5$ semanas entre as pacientes sem trombofilias e de 35,3 $\pm 5,0$ semanas entre aquelas com trombofilias. Foi evidenciado que 9 pacientes ( $45 \%$ ) tiveram partos antes da $37^{a}$ semanas de gestação, sendo $4(36,3 \%)$ entre as pacientes com trombofilias e $5(55,5 \%)$ entre aquelas sem trombofilias. Desses, dois foram indicados por trabalho de parto prematuro, quatro por sofrimento fetal, um por placenta prévia em trabalho de parto, um por doença materna grave e um por óbito fetal.

O peso dos recém-nascidos foi em média de 2.417,3 3 666,2 g e o índice Apgar de 1 e 5 minutos foi abaixo de 7 em $5 \%$ dos partos. Em $75 \%$ dos casos, a via de parto foi cesariana, sendo as indicações: cicatriz uterina prévia $(5 / 20-25 \%)$, iteratividade $(3 / 20-15 \%)$, apresentação pélvica $(3 / 20-15 \%)$, placenta prévia $(3 / 20-15 \%)$ e sofrimento fetal $(1 / 20-5 \%)$. $\mathrm{O}$ exame anatomopatológico da placenta mostrou áreas de infarto viloso em $6 / 20$ pacientes $(30 \%)$ e hematomas subcoriônicos em $4 / 20$ pacientes (20\%). O peso médio da placenta foi de $477,3 \pm 148,5 \mathrm{~g}$.

Não houve nenhuma complicação intraoperatória entre as pacientes incluídas. A média de dias de internação

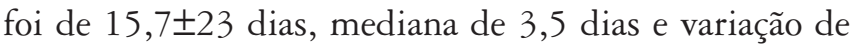
3 a 98 dias. As internações prolongadas foram devidas à alteração de vitalidade fetal (oligoâmnio ou índice de líquido amniótico reduzido) em três casos, placenta prévia em três casos e pré-eclâmpsia superajuntada em um caso.

Todas as pacientes incluídas fizeram uso de enoxaparina $(18 / 20-90 \%)$ ou heparina não fracionada $(2 / 20-10 \%)$ subcutânea durante toda gestação, sendo 14 delas na dose profilática ( 40 a $80 \mathrm{mg} /$ dia de enoxaparina ou 15.000 UI ao dia de HNF) e 6 delas na dose plena de enoxaparina ( $1 \mathrm{mg} / \mathrm{kg} 2$ vezes ao dia). O aumento da dose de heparina foi baseado na piora da dopplervelocimentria ou vitalidade fetal. O ácido acetilsalicílico (AAS) na dose de $100 \mathrm{mg} /$ dia foi utilizado em 14 gestantes durante o pré-natal e puerpério. Nenhum evento adverso relacionado ao uso dessas medicações foi observado.

\section{Discussão}

As trombofilias consistem na predisposição genética ou adquirida à trombose. Na gestação, sabe-se que a presença de trombofilias pode ser identificada em 20 a $50 \%$ das gestantes que apresentaram um evento de tromboembolismo venoso na gestação ou puerpério e tem sido cada vez mais estudada como fator causal de resultados adversos gestacionais, como trombose placentária, formas graves de doença hipertensiva específica da gestação, descolamento prematuro de placenta, restrição de crescimento fetal intrauterino e óbito fetal ${ }^{4}$.

O óbito fetal é uma complicação da gestação que traz à mulher transtornos clínicos, emocionais e obstétricos, tendo uma incidência que varia de 1,6 a 32,7 para cada 1.000 nascimentos. A etiologia do óbito fetal é indefinida em até $12 \%$ dos casos e pode ser relacionada à presença de trombofilias ${ }^{7}$.

Nossos resultados mostraram que, entre as pacientes com antecedentes de perda fetal após a $20^{\mathrm{a}}$ semana, houve uma frequência de óbito fetal de repetição de $3,7 \%$. Ouyang et al. ${ }^{19}$, em 2013, em um estudo que incluiu 61.780 mulheres, observaram uma recorrência de óbito fetal de 4,6\% e uma incidência global de um único óbito fetal de $2 \%$. Esses resultados mostraram-se compatíveis com os encontrados na nossa amostragem.

Encontramos uma frequência de trombofilias adquiridas ou hereditárias em 11 das 20 pacientes incluídas (55\%), sendo 3 diagnosticadas como deficiência de proteína $S$ (confirmada no puerpério), 7 como síndrome antifosfolípide e 1 como mutação do gene da protrombina na forma heterozigota. A rara prevalência das trombofilias genéticas na população dificulta o estudo adequado de sua relação com o óbito fetal de repetição.

$\mathrm{Na}$ literatura, um estudo caso-controle controlado incluiu 232 pacientes que foram submetidas à pesquisa de trombofilias após uma perda fetal tardia ${ }^{20}$. Dois ou mais óbitos fetais foram encontrados em $36,2 \%$ dos casos. Dentre esse grupo, $10,7 \%$ apresentavam deficiência de proteína S e 1,2\%, mutação da protrombina. Não houve pacientes com as demais trombofilias pesquisadas. A presença de deficiência de proteína $S$ foi fator de risco significativo para perda fetal de repetição, quando comparada a apenas 1 óbito fetal $(10,7$ versus $1,4 \%, p=0,0013)$. Comparadas às pacientes saudáveis, a deficiência de proteína $\mathrm{S}$ e a presença de anticorpos anticardiolipina da classe $\mathrm{IgG}$ foram fatores de risco significativos de óbito fetal de repetição, o que é compatível com nossos resultados.

Uma meta-análise de $2003^{15}$ também mostrou que o óbito fetal recorrente acima de 22 semanas foi relacionado à presença do fator $\mathrm{V}$ de Leiden (OR 7,8; IC95\% $2,8-21,6$ ), protrombina mutante ( 5 estudos; $n=1.539$; 
OR 2,9; IC95\% 1,5 - 5,7) e deficiência de proteína S (2 estudos; $\mathrm{n}=624$; OR 14,7; IC95\% 0,9-218).

A perda fetal em pacientes com trombofilia poderia ser explicada pela formação excessiva de trombos venosos intraplacentários e infartos placentários, levando secundariamente a uma insuficiência placentária. No entanto, outros mecanismos fisiopatológicos devem estar envolvidos, uma vez que é possível ocorrer resultados gestacionais adversos mesmo na ausência de trombose placentária ${ }^{15}$.

A plausibilidade biológica do benefício do uso de agentes antitrombóticos é baseada na presumida fisiopatologia da associação de trombofilias e resultados adversos gestacionais. O mecanismo de ação da heparina é, em sua maior parte, atribuído às suas propriedades anticoagulantes, que poderiam reduzir a trombose na microvasculatura placentária. Além disso, os estudos in vitro têm mostrado que a heparina apresenta também uma ação anti-inflamatória, resultante do bloqueio das proteínas de adesão, promoção da diferenciação do trofoblasto e redução da ligação de autoanticorpos antifosfolípides às células trofoblásticas ${ }^{21}$.

O efeito da aspirina para aumentar a taxa de sucesso das gestações já não é tão claro. Todavia, conjectura-se que o efeito antiagregante dessa medicação poderia, de forma análoga, reduzir a coagulação intraplacentária e melhorar os resultados perinatais ${ }^{21}$.

Todas as pacientes com óbito de fetal de repetição em nosso serviço receberam acompanhamento rigoroso de pré-natal. Dessas, 30\% receberam exclusivamente heparina (heparina não fracionada ou de baixo peso molecular) subcutânea e $70 \%$ receberam enoxaparina associada ao AAS $100 \mathrm{mg}$ ao dia. Complicações obstétricas ocorreram em $75 \%$ das pacientes incluídas, sendo uma delas um óbito fetal na $20^{a}$ semana de gestação.

Uma meta-análise de 2013 mostrou que ainda há pouca evidência do uso de heparina e AAS em gestantes trombofílicas com perdas fetais ${ }^{21}$. Os resultados de diversos estudos pequenos, retrospectivos e prospectivos em mulheres com trombofilias hereditárias, com ou sem complicações gestacionais anteriores, sugerem um benefício da terapia antitrombótica para reduzir complicações obstétricas. No entanto, esses trabalhos são heterogêneos entre si. Em pacientes com síndrome antifosfolípide, o uso da heparina de baixo peso molecular combinada com o uso da aspirina parece beneficiar aquelas com perdas fetais de repetição ou óbitos fetais de segundo trimestre.

Em um ensaio clínico com pacientes trombofílicas, portadoras da mutação do fator $\mathrm{V}$ de Leiden, mutação da protrombina ou deficiência de proteína $S$, com antecedente de pelo menos um óbito fetal acima de 12 semanas, o uso de enoxaparina $40 \mathrm{mg}$ por dia $(n=80)$ aumentou a taxa de nativivos quando comparadas às pacientes randomizadas para receber AAS $100 \mathrm{mg}$ por dia (86 e 29\%, respectivamente; redução de risco absoluto de 57; OR 15,5; IC95\% $7-34)^{22}$.

$\mathrm{Na}$ nossa casuística de pacientes com mau passado obstétrico, que apresentaram óbitos fetais de repetição, os resultados gestacionais foram favoráveis em $95 \%$ das pacientes. Entre pacientes estudadas, $75 \%$ apresentaram complicações obstétricas, sendo 7 (35\%) entre aquelas com trombofilias identificadas versus $8(40 \%)$ entre as pacientes sem trombofilias. A presença de hematomas subcoriônicos ou infartos placentários foi de 5 (25\%) naquelas com trombofilias versus $3(15 \%)$ naquelas sem trombofilias.

Nosso estudo permitiu selecionar um grupo restrito de pacientes, com complicações obstétricas graves e repetidas que têm grande impacto na saúde física e psicológica das mulheres e que trazem grande inquietação ao obstetra. Os resultados sugerem que a pesquisa de trombofilias deve ser realizada em todas as gestantes com óbitos fetais de repetição a partir da $20^{a}$ semana de gestação, como forma de identificar possíveis fatores causas passíveis de tratamento clínico e farmacológico. O uso da terapia antitrombótica nesses casos ainda tem pouca evidência na literatura; no entanto, nosso trabalho sugere que o seu emprego pode implicar melhores resultados obstétricos e maior taxa de nascidos vivos.

\section{Referências}

1. Baptista FS, Oliveira ALML. Trombofilias. In: Zugaib M, Bittar RE. Protocolos assistenciais: Clínica Obstétrica FMUSP. 3a ed. São Paulo: Atheneu; 2008. p. 193-204.

2. Battinelli EM, Baver KA. Thrombophilias in pregnancy. Hematol Oncol Clin North Am. 2011 ;25(2):323-33.

3. Ruano R, Yoshizaki CT, Martinelli S, Pereira PP. Doenças tromboembólicas. In: Zugaib $M$, editor. Zugaib obstetrícia. 2a ed. São Paulo: Manole; 2012. p. 773-89.
4. Gerhardt A, Scharf RE, Beckmann MW, Struve S, Bender HG, Pillny $M$, et al. Prothrombin and factor $V$ mutations in women with a history of thrombosis during pregnancy and the puerperium. $N$ Engl J Med. 2000;342(6):374-80.

5. Zahed LF, Rayes RF, Mahfouz RA, Taher AT, Maarouf HH, Nassar AH. Prevalence of factor $V$ Leiden, prothrombin and methylene tetrahydrofolate reductase mutations in women with adverse pregnancy outcomes in Lebanon. Am J Obstet Gynecol. 2006;195(4): $1114-8$. 
6. Robertson L, Wu O, Langhorne $\mathrm{P}$, Twaddle $\mathrm{S}$, Clark $\mathrm{P}$, Lowe GD, et al. Thrombophilia in pregnancy: a systematic review. $\mathrm{Br} J$ Haematol. 2006;132(2):171-96.

7. Alfirevic Z, Roberts D, Martlew V. How strong is the association between maternal thrombophilia and adverse pregnancy outcome? A systematic review. Eur J Obstet Gynecol Reprod Biol. 2002;101(1):6-14.

8. Alfirevic Z, Mousa HA, Martlew V, Briscoe L, Perez-Casal M, Toh $\mathrm{CH}$. Postnatal screening for thrombophilia in women with severe pregnancy complications. Obstet Gynecol. 2001 ; 97(5 Pt 1):753-9.

9. Paidas $\mathrm{W}, \mathrm{Ku} \mathrm{DH}$, Arkel YS. Screening and management of inherited thrombophilias in the setting of adverse pregnancy outcome. Clin Perinatol. 2004;31(4):783-805.

10. Ananth CV, Wilcox AJ. Placental abruption and perinatal mortality in the United States. Am J Epidemiol. 2001;153(4):332-7.

11. Bittar RE, Pereira PP, Liao AW. Óbito fetal. In: Zugaib M, editor. Zugaib obstetrícia. 2a ed. São Paulo: Manole; 2012. p. 749-54.

12. Greb AE, Pauli RM, Kirby RS. Accuracy of fetal death reports: comparison with data from an independent stillbirth assessment program. Am J Public Health. 1987;77(9):1202-6.

13. Rubens CE, Victora CG, Gravett MG, Nunes TM. Global report on preterm birth \& stillbirth: the foundation for innovative solutions and improved outcomes. BMC Pregnancy Childbirth. 2010;10 Suppl 1.

14. Magriples U, Ozcan T, Karne A, Copel JA. The effect of anticoagulation on antenatal ultrasound findings in pregnant women with thrombophilia. J Matern Fetal Neonatal Med. 2006;19(1):27-30.
15. Rey E, Kahn SR, David M, Shrier I. Thrombophilic disorders and fetal loss: a meta-analysis. Lancet. 2003;361 (9361):901-8.

16. Miyakis S, Lockshin MD, Atsumi T, Branch DW, Brey RL, Cervera $R$, et al. International consensus statement on an update of the classification criteria for definite antiphospholipid syndrome (APS). J Thromb Haemost. 2006;4(2):295-306.

17. Hézard N, Cornillet $P$, Droullé C, Guillot L, Potron G, Nguyen P. Factor V Leiden: detection in whole blood by ASA PCR using an additional mismatch in antepenultimate position. Thromb Res. 1997;88(1):59-66.

18. Bertina RM. Genetic approach to thrombophilia. Thromb Haemost. $2001 ; 86(1): 92-103$

19. Ouyang F, Zhang J, Betrán AP, Yang Z, Souza JP, Merialdi M. Recurrence of adverse perinatal outcomes in developing countries. Bull World Health Organ. 2013;91 (5):357-67.

20. Gris JC, Quéré I, Monpeyroux F, Mercier E, Ripart-Neveu S, Tailland $M L$, et al. Case-control study of the frequency of thrombophilic disorders in couples with late foetal loss and no thrombotic antecedent-the Nîmes Obstetricians and Haematologists Study5 (NOHA5). Thromb Haemost. 1999;81(6):891-9.

21. de Jong PG, Goddijn M, Middeldorp S. Antithrombotic therapy for pregnancy loss. Hum Reprod Update. 2013;19(6):656-73.

22. Gris JC, Mercier E, Quéré I, Lavigne-Lissalde G, Cochery-Nouvellon $E$, Hoffet $M$, et al. Low-molecular-weight heparin versus lowdose aspirin in women with one fetal loss and a constitutional thrombophilic disorder. Blood. 2004;103(10):3695-9. 